\title{
REDUCED COMPLEXITY VLC SEQUENCE DECODER
}

\author{
Hang NGUYEN, Pierre DUHAMEL \\ Alcatel, Research and Invovation, Route de Nozay, F91460, FRANCE (hang.nguyen@ alcatel.fr) \\ CNRS/LSS, Supelec, Plateau de Moulon, F92190, FRANCE (pierre.duhamel@1ss.supelec.fr)
}

\section{A. Basic ideas of the proposed reduced complexity algorithm}

The proposed decoding algorithm benefits from all the constraints that can be expressed on the whole sequence. It uses the notion of "survivor sequence" as in the Viterbi algorithm (VA), and applies it to variable length code decoding. The survivor selection takes into account the conventional Viterbi metric, the VLC structure and the source semantics constraints on the sequence. However, the straightforward application of VA meets two major difficulties: the variable length structure of the code and the additional sequence constraints. The first difficulty is resolved by the recursive construction of the lists, in which, the candidate sequences are classified by their lengths in bits. Only candidate sequences of same length in bits are considered for the survivor selection. Hence, there are one or more survivors for each possible length in bits. The second difficulty is resolved by the additional steps related to the number of DCT coefficients $r$ associated to the candidate sequences [1]. Only candidate sequences of same length in bits and same associated value $r$ are considered for the survivor selection. There is one survivor for each possible value of $r$. Hence, for each possible length in bits, there are several survivors corresponding to different possible values of $r$. This new decoder can deliver optimum hard-output values in the maximum likelihood (ML) sense, with linear complexity with the number of VLC codewords. The complexity and processing costs are similar to those of the other existing methods for decoding VLC sequences, which only make use of a partial redundancy. Another advantage of the method is that the decoded sequence is always a feasible VLC sequence of a valid image block.

However, the above mentioned additional steps, which are introduced for keeping the optimality of the algorithm when the "run" constraint is used, makes the algorithm much more complex. The basic idea of this paper is to withdraw these additional steps. Although the proposed reduced-complexity decoding algorithm is theoretically suboptimal, the hard-output sequence still meets all constraints and is among the sequences which have the highest likelihood. The (slightly) sub-optimality of the proposed reduced-complexity algorithm can be theoretically analyzed.

\section{B. Simulation results}

In Fig.1, the proposed reduced complexity decoder using the VLC structure projection and intrinsic image properties has been evaluated and compared with the conventional prefix-based VLC decoder, and the existing decoder using only the projection on the VLC structure[2-6], and the optimal decoder of paper [1].
The performance metric is chosen as the image block error rate, defined as follows:

$$
I B E R=\frac{\text { number of blocks that are erroneously decoded }}{\text { number of transmitted blocks }}
$$

A set of image blocks from three classical video sequences ("Mother-daughter", "Foreman", "Irene") is used for simulation. The video encoder under consideration is H.263. This set of image blocks are transmitted over the Gaussian (AGWN) channel with a BPSK modulation, and then decoded with the algorithms to be compared. The input of the VLC sequence decoder are soft values. The two performance plots corresponding to the proposed reduced complexity algorithm and the optimal algorithm of paper [11] are superposed. Hence, the performance of the proposed reduced complexity algorithm is similar to the one of the optimal decoder proposed in paper [11]. The proposed reduced complexity algorithm is only slightly suboptimal. The complexity of the two algorithms can be experimentally evaluated by storing the number of sequences in each list $L_{k}$. Our simulation shows that each list $L_{k}$ contains on average 20 sequences for the optimal algorithm of paper [11] and only 4 for the proposed reduced complexity algorithm. So, a gain of 5 times in terms of complexity is obtained.

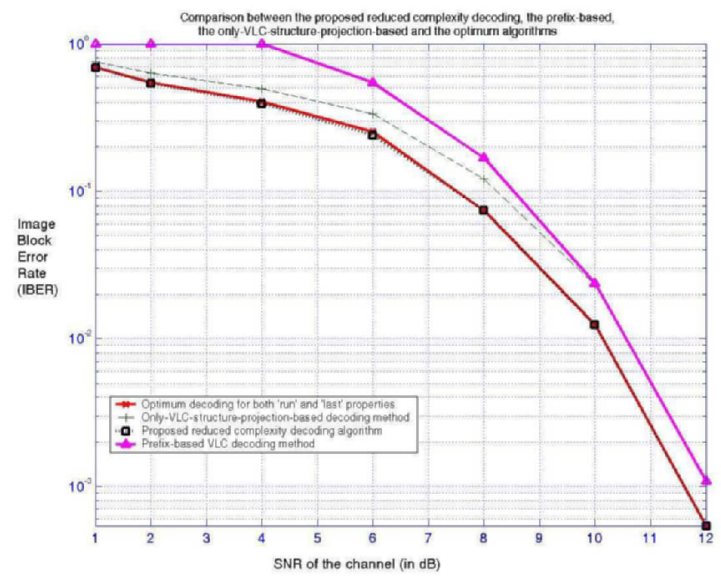

Fig. 1: Comparison between the proposed reduced-complexity decoding, the prefix-based, the only-VLC-structure-projection-based and the paper [11] optimum decoding algorithms

\section{REFERENCES}

[1] H. Nguyen, P. Duhamel, Jerome Brouet, Denis Rouffet "Optimal VLC sequence decoding exploiting additional video stream properties", IEEE ICASSP, 2004. 